Check for updates

Cite this: RSC Adv., 2017, 7, 32960

Received 28th November 2016

Accepted 16th June 2017

DOI: $10.1039 / c 6 r a 27474 a$

rsc.li/rsc-advances

\title{
Biodegradable, pH-responsive chitosan aerogels for biomedical applications
}

\author{
J. Radwan-Pragłowska, ${ }^{\text {*a }}$ M. Piątkowski, (D) ${ }^{a}$ Ł. Janus, ${ }^{a}$ D. Bogdał ${ }^{a}$ and D. Matysek ${ }^{b}$
}

Novel pH-responsive biodegradable chitosan aerogels for biomedical applications were developed. Prepared from propylene glycol and bioactive acids under MW conditions hydrogels were transformed into aerogels by lyophilisation. Obtained materials were characterized with FT-IR spectroscopy and SEM imaging. Biodegradability, bioactivity and $\mathrm{pH}$ response were analysed. An anticancer drug release profile was investigated.

Recently, the rapid development of medicine and pharmacy in such areas as tissue engineering, ${ }^{1,2}$ theranostics, ${ }^{3}$ controlled drug delivery and release systems ${ }^{4,5}$ and intelligent wound dressings ${ }^{6}$ has been observed resulting in the need for new types of biomaterials with advanced properties. Lately, our scientific team has developed a new method of chitosan hydrogel obtainment, characterized by high swelling coefficients. It was discovered that application of microwave radiation, as well as propylene glycol and an appropriate acid or amino acid in a specific ratio as crosslinking agents, results in the obtainment of a material with excellent sorption capacity. Chitosan is a polymer obtained by deacetylation of chitin known for its beneficial characteristics. ${ }^{7}$ It gained the attention of scientists in the $19^{\text {th }}$ century and properties of the polymer like antibacterial, ${ }^{7}$ antioxidant ${ }^{8}$ or hemostatic activity, ${ }^{9}$ biodegradability ${ }^{10}$ lack of toxicity and biotolerance ${ }^{11}$ have been found. Currently, it is one of the most leading macromolecular compounds in the field of biomedicine. ${ }^{12}$ Two functional groups of the polymer enable various chemical modifications resulting in the obtainment of the materials with better characteristics. These include methylation, carboxylation, alkylation, sulphonation and many others. ${ }^{13-15}$ During modifications such a parameter as deacetylation degree must be precisely measured, since it influences characteristics of the material. ${ }^{16-19}$ Recently, chitosan has been blended with other polymers, natural or synthetic, to increase its bioactivity or affinity to specified tissues. ${ }^{\mathbf{2 0 , 2 1}}$ Commonly used are poly(ethylene glycol), poly(vinyl alcohol), poly(lactic acid), polyamide- 6 or poly(vinyl pyrrolidone).$^{22-24}$ Nevertheless, such trials of some biological properties enhancement may lead to deterioration of its other features like biodegradability, antipyrogenity and cause oxidation stress after body placement.

${ }^{a}$ Department of Biotechnology and Physical Chemistry, Faculty of Chemical Engineering and Technology, Cracow University of Technology, Warszawska 24 St, Cracow, 31-155, Poland. E-mail: jrpraglowska@chemia.pk.edu.pl

${ }^{b}$ Faculty of Mining and Geology, Technical University of Ostrava, 17. Listopadu 15 St, Poruba, Ostrava, Czech Republic
Chitosan in medicine and pharmacy is mostly applied in form of physical or chemical hydrogels. ${ }^{25-30}$ Their obtainment is connected with the usage of various crosslinking agents like 1ethyl-3-(3-dimethylaminopropyl)carbodiimide (EDS) leading to fibrosis and inflammation. ${ }^{31}$ Alternatively, glutaric aldehyde or genipin were used which resulted in significant decrease of susceptibility of the material to biological degradation, hydrophilicity and adhesiveness. Application of such reagents during polymerization reactions was also associated with the presence of unreacted molecules, which when non chemically bonded resulted in toxic effect on human cells. ${ }^{32-36}$ Therefore, new crosslinking agents and reaction conditions had to be developed. A new approach of the chitosan hydrogels formation is based on crosslinkers, which are nontoxic and inert to living cells even when they are not fully incorporated into polymeric backbone. Fully biocompatible and biodegradable chitosan hydrogels have some significant limits in their applications due to their solubility in low $\mathrm{pH}$, lack of durable structure and difficulties in active substances placement and release, as well as functionality preservation in time..$^{37,38}$ Thus alternative forms of polymer or polymeric composites like nanoparticles, were developed but it strongly limited biomaterial application due to lower durability. ${ }^{39,40}$ The number of different chitosan modification methods caused an increase in requirements for biomaterials obtainment pathways, which have to be not only effective but also harmless to the environment and performed according to Green Chemistry principles. Although, there is a great number of chitosan concerning publications, no one yet has proposed biodegradable, biotolerant and bioactive chitosan aerogels with easily programmed properties obtained by rapid, efficient, eco-friendly method which has many potential biomedical applications.

Herein we report a new type of multifunctional chitosanbased aerogels obtainment method under microwave irradiation. Our innovation concerns novel approach to aerogel materials, which are applied as thermal, moisture or sound insulators and preparation of chitosan hydrogels with high 
sorption capacity using organic acids and propylene glycol as crosslinkers followed by their conversion into super light porous biomaterial by lyophilisation process. All chitosan aerogels were prepared in two-step method from chitosan flakes of the known average molar mass determined by viscosimetric measurements (401 $000 \mathrm{~g} \mathrm{~mol}^{-1}$ ) and the deacetylation degree of $79.6 \%$. All raw materials used for chitosan aerogels preparation including: L-aspartic acid, succinic and adipic acid can be produced during fermentation process of waste molasses as the main carbon source. Levulinic acid is a basic product of catalytic degradation and dehydration reaction of cellulose processing under acidic conditions.

The general procedure of chitosan aerogels preparation involves dissolving chitosan flakes $(0.5 \mathrm{~g})$ in $10 \mathrm{~mL}$ of $2 \%$ acetic acid solution, heating and mixing heterogeneous system until all chitosan flakes dissolute. Speed of chitosan dissolution depends on $\mathrm{pH}$ of the aquatic solution. Generally chitosan dissolves in water below $\mathrm{pH}=6.3$, but to shorten the dissolution time $0.1 \mathrm{~g}$ of each acid was used, as well as $1 \mathrm{~mL}$ of propylene glycol. A drug was incorporated into polymeric matrix at the end of the homogenization process (Fig. 1).

After obtainment of homogenous solution, the samples were irradiated using $400 \mathrm{~W}$ for $20 \mathrm{~min}$, frozen and freeze dried. Lyophilisation process significantly reduced mass of prepared samples. As a result of the lyophilisation process a highly porous material was obtained (Fig. 2).

Aerogels were characterized by IR spectroscopy. In the Fig. 3A FT-IR spectrum of pure chitosan is presented. Stretching vibrations of $-\mathrm{OH}$ and $-\mathrm{NH}$ groups are observed at 3276 and $3357 \mathrm{~cm}^{-1}$. At $2919 \mathrm{~cm}^{-1}$ stretching vibrations from $\mathrm{C}-\mathrm{H}$ aliphatic chains are present. At 1650 and $1637 \mathrm{~cm}^{-1}$ stretching vibrations of carbonyl group $\mathrm{C}=\mathrm{O}$ in amide group can be observed. At $1578 \mathrm{~cm}^{-1}$ bending vibrations of $-\mathrm{CH}_{3}$ groups in the acetylated units of chitosan are visible. At $1557 \mathrm{~cm}^{-1}$ a band with a low intensity characteristic for $N$-acetylated groups is present. At $1419 \mathrm{~cm}^{-1} \mathrm{a}-\mathrm{CH}_{2}$ - bending vibrations are observed in the spectrum. At $1320 \mathrm{~cm}^{-1}$ stretching vibrations from $\mathrm{C}-\mathrm{O}-\mathrm{N}$ structure and at $1251 \mathrm{~cm}^{-1}$ stretching vibrations from $\mathrm{C}-\mathrm{O}-\mathrm{C}$ bridge are observed. At $1073 \mathrm{~cm}^{-1}$ and $1030 \mathrm{~cm}^{-1}$ stretching $\mathrm{C}-\mathrm{O}$ vibrations and skeletal vibrations involving a C-O-C stretching band are present. At $895 \mathrm{~cm}^{-1}$ vibrations responsible for glycosidic bonds are observed. In the Fig. 3C a chitosan/levulinic acid aerogel spectrum is presented. The

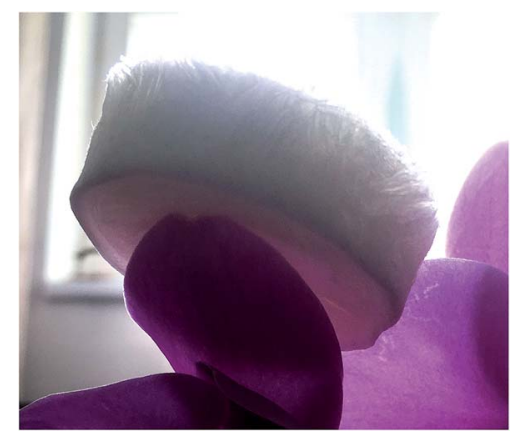

Fig. 1 Superlight chitosan aerogel with density of $0.06 \mathrm{~g} \mathrm{~cm}^{-3}$.

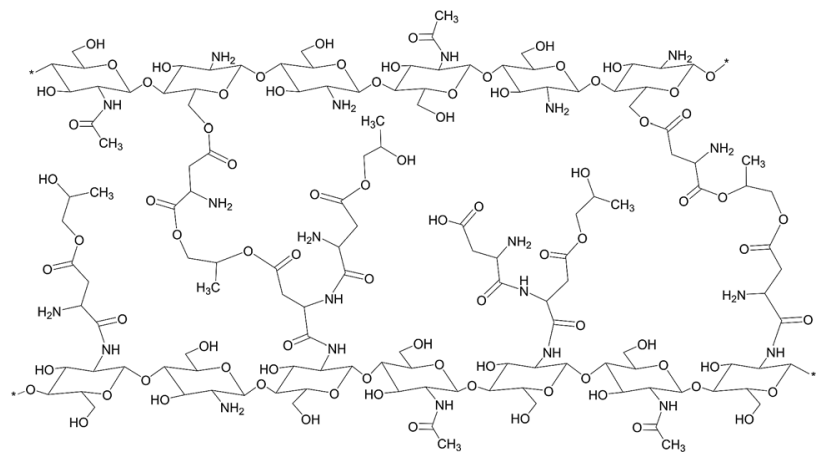

Fig. 2 Proposed chemical structure of the chitosan aerogel obtained from L-aspartic acid.

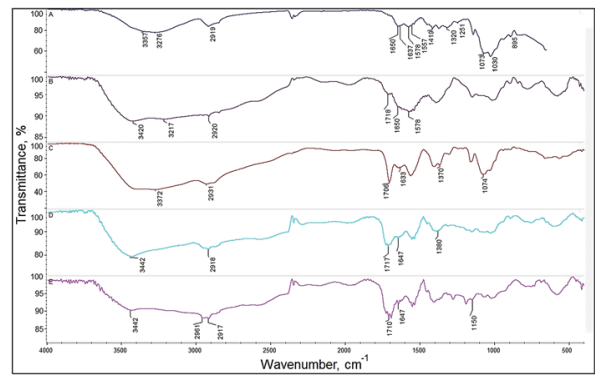

Fig. 3 Chitosan aerogels FT-IR spectra. (A) Chitosan, (B) chitosan/Laspartic acid aerogel, (C) chitosan/levulinic acid aerogel, (D) chitosan/ succinic acid aerogel, (E) chitosan/adipic acid aerogel.

main difference between chitosan and chitosan/levulinic acid aerogel is a strong peak at $1706 \mathrm{~cm}^{-1}$ from ketone group vibrations $\mathrm{C}=\mathrm{O}$ contained in levulinic acid molecule. Low intensity stretching vibrations from amide carbonyl $\mathrm{C}=\mathrm{O}$ group are still present at $1650 \mathrm{~cm}^{-1}$. At $1557 \mathrm{~cm}^{-1}$ a strong band from protonated $-\mathrm{NH}_{3}{ }^{+}$groups appears. In the Fig. 3B a chitosan/Laspartic acid aerogel spectrum is present. A strong and broad band from 2900 to $3600 \mathrm{~cm}^{-1}$ appeared which is caused by introducing to chitosan a large amount of carboxylic groups and the primary hydroxyl groups of chitosan. Hydroxyl groups of carboxylic acid $-(\mathrm{C}=\mathrm{O})-\mathrm{OH}$ and $-\mathrm{NH}$ vibrations caused a strong absorption of IR radiation in this area of spectrum.

A strong band from stretching vibrations of carbonyl groups appeared in a ragged strong peak with maximum absorption at 1729 and $1710 \mathrm{~cm}^{-1}$ in the Fig. 3E. At $1555 \mathrm{~cm}^{-1}$ again a small peak responsible for protonated amino group $-\mathrm{NH}_{3}{ }^{+}$appeared indicating that carboxylic groups of acids interacted with chitosan free amine groups and protonated it. Due to a high similarity in chemical structure of adipic and succinic acid spectrum of chitosan/adipic acid aerogel and chitosan/succinic acid aerogel (Fig. 3D) are very similar.

Also a strong band between 2400 and $3700 \mathrm{~cm}^{-1}$ caused by vibrations of $-\mathrm{OH}$ and $-\mathrm{NH}$ groups is observed. A stronger absorption at $2920 \mathrm{~cm}^{-1}$ is observed because succinic acid contains two methylene groups which contain four $\mathrm{C}-\mathrm{H}$ bonds. Again in this spectra a small peak at $1552 \mathrm{~cm}^{-1}$ indicates that protonated amino groups are present. Visible changes in 
chemical structure of the chitosan confirms chemical modification of the polymer due to the crosslinking reaction. Bands typical for amide bonds can be observed which proves the hypothetical chemical structure of the aerogel presented in the Fig. 2.

The morphology of chitosan aerogels was characterized by SEM. Chitosan aerogels obtained using L-aspartic acid characterized by a large smooth wavy, little ragged flakes having an average size of 400 to $600 \mu \mathrm{m}$. The surface of the obtained aerogel wasn't cracked. In contrast to hydrogel obtained using $\mathrm{L}^{-}$ aspartic acid chitosan aerogel synthetized in succinic acid solution (Fig. 4B) was characterized by a large morphological variation. In the picture both flat structure and ragged one in homogenous areas were visible. The size of pores in the regions of occurrence of smaller structures varied from 50 to $150 \mu \mathrm{m}$. In the picture at the ends of flakes finer structures were observed. Aerogel obtained by using levulinic acid was characterized by rather uniform wall thickness. Addition of adipic acid to chitosan creates trapezoidal slightly rolled up shapes similar to flower petals after freeze drying process. On the surface of the aerogel very thin porous structures reminiscent of the thread cobweb can be observed. The size of the largest elements seen in the picture oscillates between 200 and $400 \mu \mathrm{m}$. The sizes of visible substructures were from 5 to $15 \mu \mathrm{m}$. The aerogel obtained from levulinic acid was characterized by the most uniform pattern. The whole aerogel surface was filled up with similar size pores in the range from 70 to $150 \mu \mathrm{m}$. The wall thickness of aerogel was also uniform.

The biodegradation degree of the chitosan aerogels was measured. The biodegradation study was conducted according to OECD $301 \mathrm{~B}: \mathrm{CO}_{2}$ evolution test. Firstly, the amount of Total Organic Carbon (TOC) in aerogels samples was determined.

The activated sludge was added to a mineral medium to reach the concentration $20 \mathrm{mg} \mathrm{L}^{-1}$ of suspended solids. To the $2 \mathrm{~L}$ of this solution chitosan aerogels were added to reach the concentration of TOC at the level of $20 \mathrm{mg} \mathrm{L}^{-1}$. During the study also a blank solution was prepared. The level of released carbon dioxide was monitored every 2-5 days. Based on the amount of
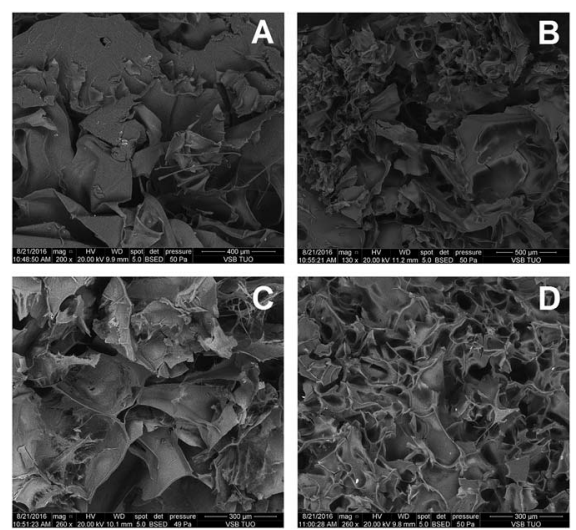

Fig. 4 SEM images of four prepared chitosan aerogels. (A) Chitosan/Laspartic aerogel structure, (B) chitosan/succinic acid aerogel structure, (C) chitosan/adipic acid aerogel structure, (D) chitosan/levulinic acid aerogel structure. carbon dioxide released by samples biodegradation degree was calculated. In the Fig. 5 biodegradation process progress is presented. During first 1-4 days of biodegradation process (lag phase) microorganisms were adapting to a new source of carbon which was contained in chitosan polymer and added organic acids. The adaptation process depends on the chemical structure of biodegradable material, the medium on which microorganisms were previously multiplicated, the physical form of the material, temperature and mineral composition of the media. After the adaptation process a logarithmic phase of biodegradation process started. During this phase a lot of carbon dioxide was released to the atmosphere and the amount of total organic carbon level decreased. From day 5 to 10 organic matter was over $80 \%$ fully degraded. After day 11 a plateau phase was observed. The end of carbon dioxide release means that all matter capable of biodegradation process was consumed by microorganisms.

To examine the behaviour of prepared chitosan aerogels in conditions imitating human organism incubation tests in Ringer and SBF fluid were performed. Ringer solution contained: $6.8 \mathrm{~g} \mathrm{~L}^{-1} \mathrm{NaCl}, 0.3 \mathrm{~g} \mathrm{~L}^{-1} \mathrm{KCl}$ and $0.48 \mathrm{~g} \mathrm{~L}^{-1} \mathrm{CaCl}_{2} \cdot 2 \mathrm{H}_{2} \mathrm{O}$. The $\mathrm{pH}$ of the prepared solution was 6.05. Simulated body fluid solution contained: $8.00 \mathrm{~g} \mathrm{~L}^{-1} \mathrm{NaCl}, 0.35 \mathrm{~g} \mathrm{~L}^{-1} \mathrm{NaHCO}_{3}, 0.22 \mathrm{~g}$ $\mathrm{L}^{-1} \mathrm{KCl}, 0.23 \mathrm{~g} \mathrm{~L}^{-1} \mathrm{KH}_{2} \mathrm{PO}_{4} \cdot 3 \mathrm{H}_{2} \mathrm{O}, 0.31 \mathrm{~g} \mathrm{~L}^{-1} \mathrm{MgCl}_{2} \cdot 6 \mathrm{H}_{2} \mathrm{O}$, $0.28 \mathrm{~g} \mathrm{~L}^{-1} \mathrm{CaCl}_{2}, 0.07 \mathrm{~g} \mathrm{~L}^{-1} \mathrm{Na}_{2} \mathrm{SO}_{4}$ and $\mathrm{HCl}$ to reach $\mathrm{pH}=7.25$. To a $15 \mathrm{~mL}$ of prepared fluid $0.5 \mathrm{~g}$ of aerogel was added. The samples were incubated in $37^{\circ} \mathrm{C}$. During 8 days change of $\mathrm{pH}$ of the prepared solutions was monitored. The results are shown in the Fig. 6 and in the 7 .

In the case of all chitosan aerogels samples no significant changes in $\mathrm{pH}$ values were observed that could suggest important changes in biomaterials chemical composition or morphological condition. Aerogels obtained from levulinic, $\mathrm{L}^{-}$ aspartic, adipic and succinic acid can be considered as inert to biological fluids according to examination results. Carboxyl groups resulted in hydrolysis could lower $\mathrm{pH}$ value due to dissociation (adipic, succinic). In the case of levulinic and aspartic acid stronger impact on body environment connected with faster polymeric matrix natural degradation can be

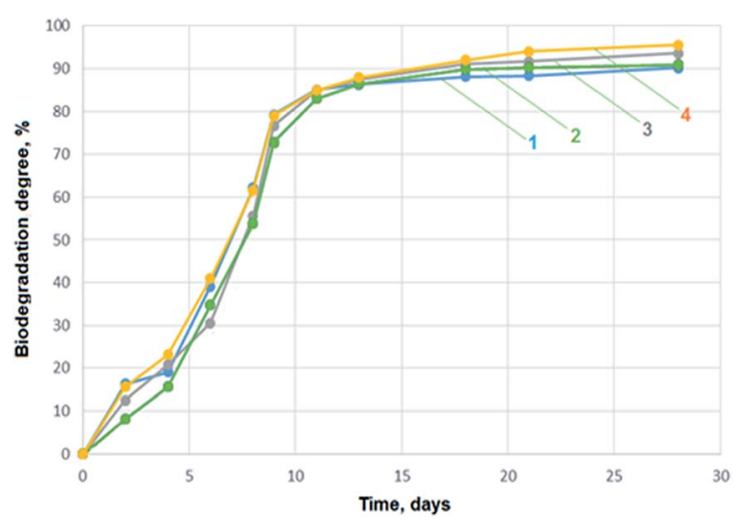

Fig. 5 The results of the chitosan/organic acid aerogel biodegradation study. (1) Chitosan/L-aspartic acid aerogel, (2) chitosan/levulinic acid aerogel, (3) chitosan/adipic acid aerogel, (4) chitosan/succinic acid aerogel. 


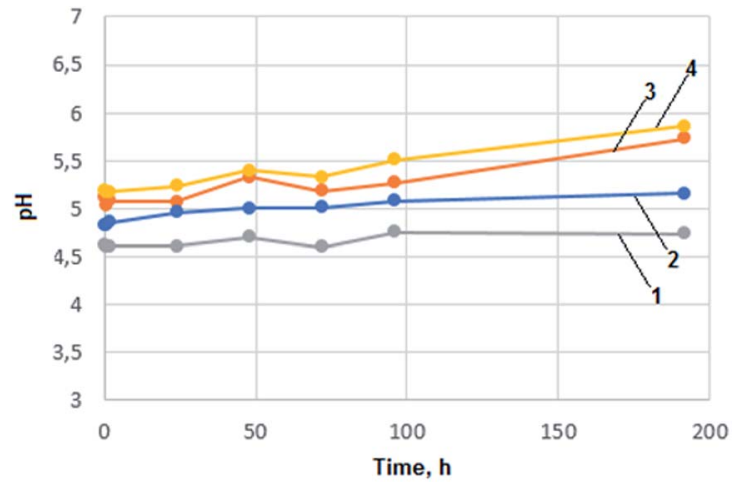

Fig. 6 Incubation study of chitosan aerogels in Ringer solution. (1) Chitosan/succinic acid aerogel, (2) chitosan/adipic acid aerogel, (3) chitosan/levulinic acid aerogel, (4) chitosan/L-aspartic acid aerogel.

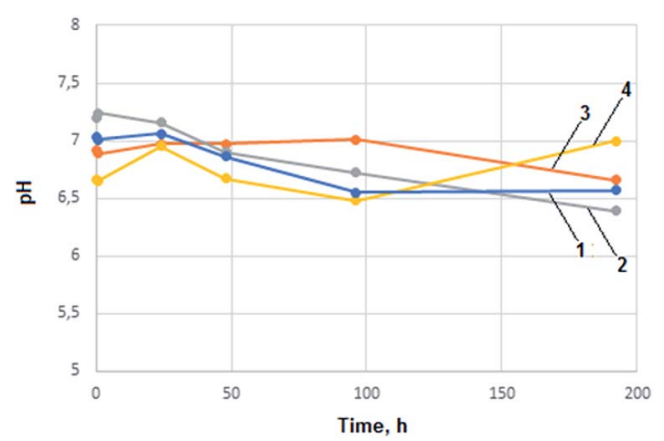

Fig. 7 Incubation study of chitosan aerogels in SBF solution. (1) Chitosan/adipic acid aerogel, (2) chitosan/succinic acid aerogel, (3) chitosan/levulinic acid aerogel, (4) chitosan/L-aspartic acid aerogel.

observed. An increase in $\mathrm{pH}$ value resulting from $-\mathrm{NH}_{2}$ groups protonation in the case of $\mathrm{L}$-aspartic acid can be noticed. $\mathrm{pH}$ increases in the case of levulinic acid was caused by ionexchange process.

A drug release profile was investigated (Fig. 8). As a model substance 5 -fluorouracil was used. The anticancer drug was incorporated into the polymeric matrix after dissolution of chitosan in slightly acidic solution. A homogenous yellow/ orange solution was obtained. The samples after drug addition and homogenization were freeze dried. The concentration of active substance was $50 \mathrm{mg} \mathrm{g}^{-1}$ of polymer matrix. Three solvents, $50 \mathrm{~mL}$ each, were used: $0.1 \mathrm{M}$ solution of hydrochloric acid, distilled water and hexane. To each solvent $0.20 \mathrm{~g}$ of dry aerogel were added and the mixtures were stirred for several hours. The hydrochloric acid solution had the strongest degradation power to aerogel. After $70-80 \mathrm{~min}$ an equilibrium state was reached for all aerogels in acidic solution. The highest amount of released 5-fluorouracil $(8.2 \mathrm{mg})$ to acid solution for chitosan/adipic acid aerogel was observed and the lowest for chitosan/levulinic acid aerogel $(6.7 \mathrm{mg})$. During the first 20 minutes over $60 \%$ of drug for all aerogels samples was released to hydrochloric acid solution. Distilled water did not have such high destructive properties to aerogels and the amount of released 5-fluorouracil was significantly lower. To dissolve chitosan in water a high hydronium ion concentration is required
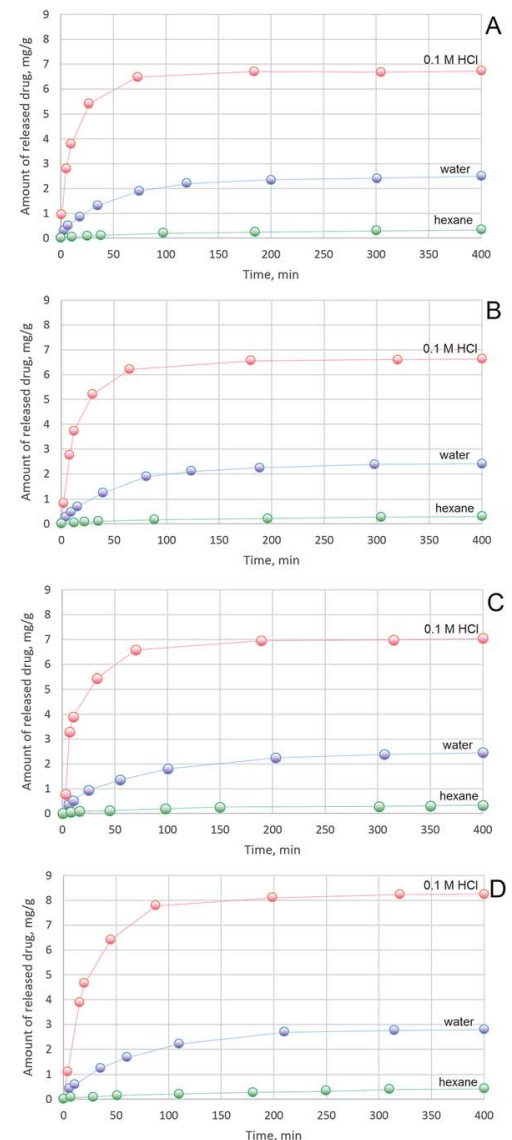

Fig. 8 5-Fluorouracil release profile from aerogels to $0.1 \mathrm{M} \mathrm{HCl}$ solution, distilled water and hexane from (A) chitosan/L-aspartic acid aerogel, (B) chitosan/succinic acid aerogel, (C) chitosan/levulinic acid aerogel, (D) chitosan/adipic acid aerogel.

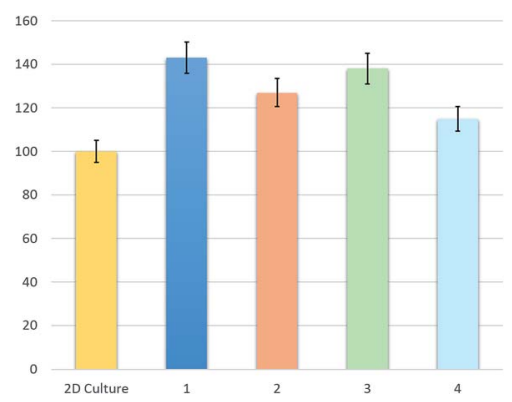

Fig. 9 Proliferation study of chitosan aerogels. 2D culture - reference sample, (1) chitosan/L-aspartic acid aerogel, (2) chitosan/succinic acid aerogel, (3) chitosan/adipic acid aerogel, (4) chitosan/levulinic acid aerogel.

to protonate free primary amine groups $\left(\mathrm{R}-\mathrm{NH}_{3}{ }^{+}\right)$. Amine groups present on chitosan chain are protonated and a stable polycationic lipophilic colloid is formed. $2.4 \mathrm{mg}$ of drug for chitosan/levulinic acid aerogel was released, 2.5, 2.8 and 2.4 for chitosan/succinic acid, adipic and aspartic acid aerogel respectively. To simulate the lipophilic environment a drug release of 5 -fluorouracil to hexane was investigated. The amount of released 5 -fluorouracil was very low in range $0.30-$ 
0.45 for all examined aerogels. Since 5 -fluorouracil is more soluble in $0.1 \mathrm{M} \mathrm{HCl}$ than in water some additional tests were performed to confirm the aerogels' $\mathrm{pH}$ sensitivity. Anticancer drug was replaced by a bromocresol red. Controlled dye release study in $0.1 \mathrm{M} \mathrm{HCl}$ aquatic solution as well as in distilled water were performed. The results were analogical which proves that differences in substance release profile are correlated with the degradation of the polymeric matrix in acidic $\mathrm{pH}$. To determine bioactivity of the chitosan aerogels a proliferation study was performed. Cell culture was proceeded using L929 fibroblasts cultured in DMEM medium supplemented and 1\% antibiotic/ antimycotic solution with $10 \%$ fetal bovine. All tests were carried out at $37{ }^{\circ} \mathrm{C}$ in $5 \% \mathrm{CO}_{2}$. The humidity was $95 \%$. Proliferation investigation was performed for five days applying cultures of $95 \%$ viability. The cells viability was estimated by trypan blue dye exclusion assay. Proliferation of fibroblasts was determined by MTT assay. As a control a plastic plate was used. In the Fig. 9 results of proliferation study are presented. None of the samples were cytotoxic. All four samples had a positive effect cells proliferation activity in comparison with reference sample. The best results were observed for the aerogel prepared from chitosan and L-aspartic acid which enhanced fibroblasts proliferation by $43 \%$. It can be caused by the fact that this aerogel mimicked extracellular matrix in the best way due to its chemical composition. Also L-aspartic acid-based aerogel had the highest surface area which could provide best nutrients and oxygen delivery and enabled cells anchorage. Similar results were obtained for the aerogel of alike morphology prepared from adipic acid which enhanced proliferation of the fibroblasts by $38 \%$. The weakest proliferation accelerating effect was observed for the aerogel of the smallest pores diameter (aerogel from levulinic acid). Pure chitosan is rich in free amino groups, which can strongly interact with fibroblast by electrostatic interactions thus limiting their proliferation activity. Chemical modification of the biopolymer with amino acids caused the significant reduction of the number of $-\mathrm{NH}_{2}$ groups what could enhance cells growth without changing its lack of cytotoxicity.

\section{Conclusions}

In summary we have developed a new, MW-assisted eco-friendly method of biodegradable, $\mathrm{pH}$ sensitive chitosan aerogels synthesis that may have various applications in medicine and pharmacy. Novel biomaterials were obtained by a two-step process comprising hydrogel obtainment under microwave irradiation and its lyophilisation. As crosslinking agents nontoxic substances were applied such as biotolerant acids and propylene glycol. Application of various acids enabled obtainment of the 3D structure with different size, shape and distribution of formed pores. Ready aerogels were fully biodegradable and of slightly different bioactivity. Biomaterials had a positive impact on proliferation activity. In comparison with 2D culture proliferation was enhanced up to $43 \%$. Chitosan-based biomaterials were investigated for their application in controlled drug release system for anti-cancer drugs. Their behaviour was analysed in environments of different $\mathrm{pH}$ value and polarity. To explain the difference between release profiles of active substance a chemical structure analysis of polymer must be done. A chitosan unit contains one polar primary hydroxyl group, one secondary hydroxyl group and one oxygen atom in the ring. The units are coupled with glycosidic bonds. Additionally, chitosan aerogels contain organic acids which makes the polymer material highly polar. The weak interactions between two phases, low ability to penetrate deep into the material and adsorption properties of chitosan prevent drug molecules leaving from aerogel material. Development of aerogels synthesis method enabled obtainment of polymeric matrixes with dedicated and programmed properties. Their further modification will be a subject of the future research and publications.

\section{Notes and references}

1 R. Logith Kumar, A. KeshavNarayan, S. Dhivya, A. Chavla, S. Saravanan and N. Selvamurugan, Carbohydr. Polym., 2016, 151, 172-188.

2 F. Croisier and C. Jérôme, Eur. Polym. J., 2013, 49, 780-792.

3 H. Min, D. You, S. Son, S. Jeon, J. Park, S. Lee, I. Kwon and K. Kim, Theranostics, 2015, 5, 1402-1418.

4 A. Islam, M. Riaz and T. Yasin, Int. J. Biol. Macromol., 2013, 59, 119-124.

5 Q. Yuan, J. Shah, S. Hein and R. Misra, Acta Biomater., 2010, 6, 1140-1148.

6 S. Ahmed and S. Ikram, Achiev. Life Sci., 2016, 10, 27-37.

7 M. Moreno-Vásquez, E. Valenzuela-Buitimea, M. PlascenciaJatomea, J. Encinas-Encinas, F. Rodríguez-Félix, S. SánchezValdes, E. Rosas-Burgos, V. Ocaño-Higuera and A. Graciano-Verdugo, Carbohydr. Polym., 2017, 155, 117-127. 8 M. Kaya, T. Baran, M. Asan-Ozusaglam, Y. Cakmak, K. Tozak, A. Mol, A. Mentes and G. Sezen, Biotechnol. Bioprocess Eng., 2015, 20, 168-179.

9 M. Pogorielov and V. Sikora, Eur. J. Med. B, 2015, 2, 24-33.

10 H. Zheng, C. Tang and C. Yin, Biomaterials, 2015, 70, 126137.

11 J. Yang, J. Chena, D. Pan, Y. Wan and Z. Wang, Carbohydr. Polym., 2013, 92, 719-725.

12 A. Rafique, K. Zia, M. Zuber, S. Tabasum and S. Rehman, Int. J. Biol. Macromol., 2016, 87, 141-154.

13 V. Mourya and I. Inamdar, React. Funct. Polym., 2008, 68, 1013-1051.

14 H. Abdul Khalil, C. Saurabh, A. Adnan, M. Nurul Fazita, M. Syakir, Y. Davoudpour, M. Rafatullah, C. Abdullah, M. Haafiz and R. Dungani, Carbohydr. Polym., 2016, 150, 216-226.

15 R. Belalia, S. Grelier, M. Benaissa and V. Coma, J. Agric. Food Chem., 2008, 56, 1582-1588.

16 B. Ding, H. Gao, J. Song, Y. Li, L. Zhang, X. Cao, M. Xu and J. Cai, ACS Appl. Mater. Interfaces, 2016, 8, 19739-19746.

17 K. Abdel-Gawad, A. Hifney, M. Fawzy and M. Gomaa, Food Hydrocolloids, 2017, 63, 593-601.

18 Y. Yuan, B. Chesnutt, W. Haggard and J. Bumgardner, Materials, 2011, 4, 1399-1416.

19 Y. Nam, W. Park, D. Ihm and M. Hudson, Carbohydr. Polym., 2010, 80, 291-295. 
20 M. Nampally, B. Moerschbacher and S. Kolkenbrock, Appl. Environ. Microbiol., 2012, 78, 3114-3119.

21 C. Sanjai, S. Kothan, P. Gonil, S. Saesoo and W. Sajomsang, Carbohydr. Polym., 2014, 15, 231-237.

22 A. Usman, K. Zia, M. Zuber, S. Tabasum, S. Rehman and F. Zia, Int. J. Biol. Macromol., 2016, 86, 630-645.

23 M. Nag, V. Gajbhiye, P. Kesharwani and N. Jain, Colloids Surf., B, 2016, 148, 363-370.

24 S. Majd, M. Khorasgani, S. Moshtaghian, A. Talebi and M. Khezri, Int. J. Biol. Macromol., 2016, 92, 1162-1168.

25 T. Wang, L. Chen, T. Shen and D. Wu, Int. J. Biol. Macromol., 2016, 93, 775-782.

26 Y. Xu, J. Han and H. Lin, Carbohydr. Polym., 2017, 156, 372379.

27 J. Wu, J. Liu, Y. Shi and Y. Wan, J. Mech. Behav. Biomed. Mater., 2016, 64, 161-172.

28 L. Gao, H. Gan, Z. Meng, R. Gu, Z. Wu, X. Zhu, W. Sun, J. Li, Y. Zheng, T. Sun and G. Dou, Colloids Surf., B, 2016, 148, 343-353.

29 S. Yu, X. Zhang, G. Tan, L. Tian, D. Liu, Y. Liu, X. Yang and W. Pan, Carbohydr. Polym., 2017, 155, 208-217.
30 H. Samanta and S. Ray, Carbohydr. Polym., 2014, 16, 109120.

31 J. Lin, S. Wen, H. Chung, W. Chen and C. Lou, Appl. Mech. Mater., 2014, 457-458, 44-48.

32 R. Muzzarelli, M. Mehtedi, C. Bottegoni, A. Aquili and A. Gigante, Mar. Drugs, 2015, 13, 7314-7338.

33 R. Muzzarelli, Carbohydr. Polym., 2009, 77, 1-9.

34 R. Pedro, C. Schmitt and M. Neumann, Carbohydr. Polym., 2016, 147, 97-103.

35 E. Azevedo and V. Kumar, Carbohydr. Polym., 2012, 90, 894900.

36 E. Mirzaei, A. Ramazani, M. Shafiee and M. Danaei, Int. J. Polym. Mater., 2013, 62, 605-611.

37 J. Lai, Int. J. Mol. Sci., 2012, 13, 10970-10985.

38 E. Azewedo, Int. J. Pharm. Pharm. Sci., 2015, 7, 8-14.

39 H. Wang, P. Agarwal, S. Zhao, R. Xu, J. Yu, X. Lu and X. He, Biomaterials, 2015, 72, 74-89.

40 A. Regiel-Futyra, M. Kus-Liśkiewicz, V. Sebastian, S. Irusta, M. Arruebo, G. Stochel and A. Kyzioł, ACS Appl. Mater. Interfaces, 2015, 7, 1087-1099. 